\title{
Monte Carlo simulation of aging phenomena in multilayer magnetic structures
}

\author{
Alexandr N. Purtov", Marina V. Mamonova, Vladimir V. Prudnikov, and Pavel V. Prudnikov \\ Omsk State University, Mira 55A, Omsk, Russia
}

\begin{abstract}
A Monte Carlo simulation of the non-equilibrium behavior of multilayer magnetic structures consisting of alternating magnetic and nonmagnetic nanolayers is realized. The calculated two-time autocorrelation function for the structure during its evolution starting from high-temperature initial state is analyzed. The analysis reveals aging effects characterized by a slowing down of correlation characteristics in the system with increase of the waiting time. The dependence of aging characteristics on thickness of ferromagnetic films is investigated. It is shown that, in contrast to bulk magnetic systems, the aging effects in magnetic superstructures arise not only at the ferromagnetic ordering temperature $T_{\mathrm{c}}$ in the films but also within a wide temperature range at $T \leq T_{\mathrm{c}}$.
\end{abstract}

\section{Introduction}

The behavior of systems with extremely slow dynamics stays one of the intriguing problems in theoretical physics [1]. It demonstrates a wide range of interesting phenomena such as aging, and violation of the fluctuation-dissipation theorem. Originally, these features were founded in complex spin glass systems [2] but different studies of these phenomena showed that the main properties of slow dynamics can be observed in simpler systems with second-order phase transition near critical point, because the evolution of such systems is characterized by large relaxation times [3].

The nanoscale periodicity in magnetic multilayer structures gives rise to the mesoscopic effects of the spatial spin correlation with the slow relaxation dynamics of magnetization accompanying the quenching of the system in the non-equilibrium state. In contrast to the bulk magnetic systems, where the slow dynamics and aging effects manifest themselves near the critical point [4], magnetic superstructures with nanoscale periodicity allow increasing the relaxation time owing to the effects related to the larger characteristic spin-spin correlation length. That is why the aging and nonergodicity effects can be experimentally observed in the multilayer $\mathrm{Co} / \mathrm{Cr}$ magnetic structure [5] within a wider temperature range as compared to that for the bulk magnetic systems.

We have performed in paper [6] a numerical Monte Carlo simulation of the non-equilibrium behavior of the multilayer $\mathrm{Co} / \mathrm{Cr} / \mathrm{Co}$ magnetic structure with the thickness of Co films equals to $N=3$ ML. Calculations of the two-time correlation functions and the staggered magnetization allow us to reveal the aging effects in the multilayer magnetic structure occurring not only in their non-equilibrium critical behavior at $T_{\mathrm{c}}$ but also within a wide range of temperatures at $T<. T_{\mathrm{c}}$. The revealed aging effects in the behavior of the magnetization relaxation for our model multilayer structure are in good agreement with the aging effects observed in experiments for the $\mathrm{Co} / \mathrm{Cr} / \mathrm{Co}$ structure [5]. The present work deals with study of dependence of aging characteristics on thickness of ferromagnetic films in the multilayer $\mathrm{Co} / \mathrm{Cu}(100) / \mathrm{Co}$ magnetic structure.

\section{Model, methods, and results}

We realize in this work a numerical Monte Carlo study of the nonequilibrium behavior of a multilayer magnetic structure (Figure 1) consisting of ferromagnetic films separated by nonmagnetic metal layer. The magnetic films have linear sizes of $L \times L \times N$ with imposed periodic boundary conditions in the film plane.

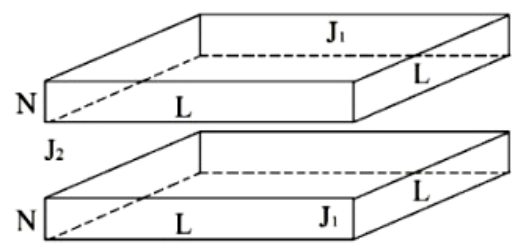

Fig. 1. Model of a multilayer structure consisting of two ferromagnetic films separated by the nonmagnetic metal film; $L$ and $N$ are the linear sizes of the films, $J_{l}$ and $J_{2}$ are the exchange integrals.

We consider the array consisting of ferromagnetic films with the thicknesses $N=3,5,7,9$ in units of monatomic layers. The exchange integral $J_{1}$ determining the interaction between the neighboring spins is assumed to be $J_{1} / k_{\mathrm{B}} T=1$ ( $k_{B}$ is the Boltzmann constant), whereas that for the interlayer interaction is $J_{2}=-0.3 J_{1}$.

* Corresponding author: alprtv@gmail.com 
The sign of $J_{2}$ is negative because the thickness of nonmagnetic spacers in multilayer structures exhibiting the giant magnetoresistance effect is tuned such that both the long-range and oscillating RKKY exchange interactions between the ferromagnetic layers effectively provide antiferromagnetism. Owing to this interaction, the magnetizations of the neighboring ferromagnetic layers have opposite orientations. Thus, the structure under study models artificially created multilayer structures exhibiting the giant magnetoresistance effect [7-9].

The magnetic characteristics of ultrathin films based on $\mathrm{Fe}, \mathrm{Co}$, and $\mathrm{Ni}$ in contact with the nonmagnetic metal substrate are most adequately described by the anisotropic Heisenberg model $[10,11]$ with the Hamiltonian

$$
H=-\sum_{<i, j>} J_{i j}\left\{\left(S_{i}^{x} S_{j}^{x}+S_{i}^{y} S_{j}{ }^{y}\right)+(1-\Delta(N)) S_{i}^{z} S_{j}^{z}\right\},
$$

where $S_{i}=\left(S_{i}{ }^{x}, S_{i}{ }^{y}, S_{i}^{z}\right)$ is a three-dimensional unit vector at the $i$-th site. $\Delta(N)$ is the parameter of anisotropy for Co films with the thickness $N(N=3,5,7,9)$ on $\mathrm{Cu}(100)$ substrate which values are presented in Table 1. The dependence of the anisotropy parameter $\Delta(N)$ on the film thickness $N$ were chosen proportional to the experimental critical temperatures for $\mathrm{Co} / \mathrm{Cu}(100)$ system [12]. The form and parameters of Hamiltonian (1) correspond to the $\mathrm{Co} / \mathrm{Cu}(100) / \mathrm{Co}$ multilayer structure, for which the ultrathin Co films exhibit the spontaneous magnetization in the $X Y$ plane of the film below the critical temperature.

Table 1. The values of the anisotropy parameter $\Delta$ and the critical temperature $T_{c}$ for films with thicknesses $N=3,5,7,9$.

\begin{tabular}{|c|c|c|}
\hline $\begin{array}{c}\text { Thickness, } \\
\mathbf{N}\end{array}$ & $\begin{array}{c}\text { Anisotropy } \\
\text { parameter, } \\
\Delta\end{array}$ & $\begin{array}{c}\text { Critical } \\
\text { temperature, } \\
\boldsymbol{T}_{\mathbf{c}}\end{array}$ \\
\hline 3 & 0.66 & $2.5413(8)$ \\
\hline 5 & 0.476 & $2.9033(12)$ \\
\hline 7 & 0.337 & $3.0356(6)$ \\
\hline 9 & 0.253 & $3.1014(11)$ \\
\hline
\end{tabular}

At the first stage of this work, we calculated the equilibrium characteristics of the multilayer structure with the aim to determine the critical temperatures $T_{\mathrm{c}}$ of the ferromagnetic films with different thicknesses $N$. For a more accurate determination of the critical temperatures, we considered the structures with films of different linear sizes $L=20,32$, and 40 . We calculated such characteristics as the staggered magnetization

$$
\mathbf{m}_{\text {stg }}=\mathbf{m}_{1}-\mathbf{m}_{2} \text {, }
$$

where $\mathbf{m}_{\mathbf{1}}$ and $\mathbf{m}_{\mathbf{2}}$ are the magnetizations of the films; staggered susceptibility

$$
\chi_{\text {stg }}=\left[<m_{s t g}^{2}>-<m_{s t g}>^{2}\right] / T N_{s},
$$

specific heat

$$
C_{h}=\left[<E^{2}>-<E>^{2}\right] / k_{B} T^{2} N_{\mathrm{s}},
$$

where $N_{s}$ is the number of spins in the film; the Binder cumulant

$$
U_{4}=\left(3-<m^{4}>/<m_{s}{ }^{2}>^{2}\right) / 2 .
$$

The values of the critical temperature $T_{\mathrm{c}}(N)$, presented in Table 1, were obtained by the method of intersection of curves for the Binder cumulant $U_{4}$ with different linear sizes $L$.

At the next stage of our studies, we considered the non-equilibrium behavior of the multilayer structure. We calculated the time dependence of magnetization for a ferromagnetic film located in the structure

$$
m(t)=\left\langle\frac{1}{N L^{2}} \sum_{i=1}^{N L^{2}} S_{i}(t)\right\rangle
$$

and the two-time autocorrelation function

$$
C\left(t, t_{w}\right)=\left\langle\frac{1}{N L^{2}} \sum_{i=1}^{N L^{2}} S_{i}(t) S_{i}\left(t_{w}\right)\right\rangle-m(t) m\left(t_{w}\right),
$$

where brackets $\langle\ldots>$ denote a statistical averaging.

The aging effects arise at $t \ll t_{\text {rel }}$, where $t_{\text {rel }}$ is the relaxation time, and manifest themselves in the dependence of the autocorrelation function on characteristic time variables such as waiting time $t_{\mathrm{w}}$ and time of observation $t-t_{\mathrm{w}}$ with $t>t_{\mathrm{w}}$ not via $t-t_{\mathrm{w}}$ only. The waiting time $t_{\mathrm{w}}$ characterizes the time interval from the preparation of the sample up to starting measurements of its characteristics. It is important that decays for the two-time autocorrelation function as function of time of observation $t-t_{\mathrm{w}}$ are slower for larger waiting times $t_{\mathrm{w}}$. Initial conditions affect the evolution of the system at $t-t_{\mathrm{w}}, t_{\mathrm{w}}<<t_{\text {rel }}$.

We considered the evolution of the system from the high-temperature initial state created at $T_{0}>>T_{c}$ with normalized staggered magnetization $m^{\text {stg }}=0.05<<1$. In calculations of the non-equilibrium behavior of the autocorrelation function $C\left(t, t_{\mathrm{w}}\right)$, we simulated the structures with the linear size of the film $L=64$, which allows us to analyze the nonequilibrium characteristics of the system at times up to 5000 Monte Carlo steps per spin $(\mathrm{MCs} / \mathrm{s})$. We used the waiting times $t_{\mathrm{w}}=10,30,50$, $100 \mathrm{MCs} / \mathrm{s}$ in the study of the two-time dependence of $C\left(t, t_{\mathrm{w}}\right)$. The averaging of $C\left(t, t_{\mathrm{w}}\right)$ was carried out on 500 $\mathrm{MC}$ runs for every $t_{\mathrm{w}}$.

The obtained time dependence of the autocorrelation function $C\left(t, t_{\mathrm{w}}\right)$ from observation time $t-t_{\mathrm{w}}$ for different $t_{\mathrm{w}}$ values and thicknesses $N$ of ferromagnetic films are presented in Figures 2 and 3 at the critical temperatures $T_{\mathrm{c}}(N)$ and in the low-temperature phase with temperatures $T_{\mathrm{c}}(N) / 2$. The curves of $C\left(t, t_{\mathrm{w}}\right)$ demonstrate the aging effects, i.e., the slowing down of time correlations with increasing system age $t_{\mathrm{w}}$. We must note that the aging effects in the multilayer structures arise not only at $T=T_{c}$ as in bulk systems, but also at $T<T_{c}$. Furthermore, the comparison of $C\left(t, t_{\mathrm{w}}\right)$ curves in Figure 2 at temperatures $T=T_{c}$ and $T_{\mathrm{c}} / 2$ shows that the aging effects become stronger in the low-temperature phase, i.e. the falling of time correlations at $T_{\mathrm{c}} / 2$ with the same waiting times $t_{\mathrm{w}}$ become more slow than at the critical 

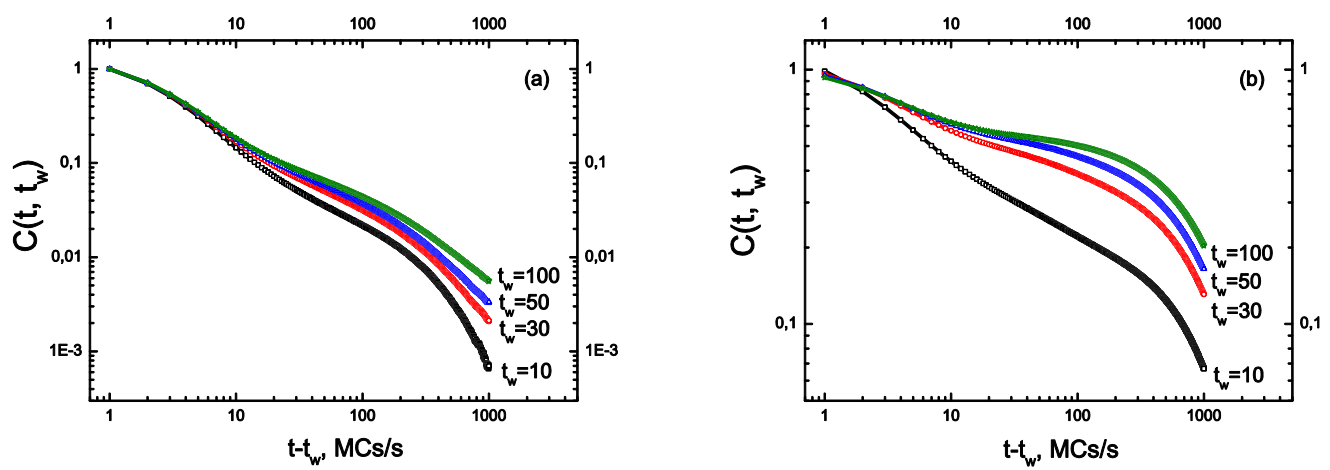

Fig. 2. Time dependence of the autocorrelation function $C\left(t, t_{w}\right)$ as a function $t-t_{w}$ for different values of $t_{\mathrm{w}}$ for $\mathrm{Co} / \mathrm{Cu}(100) / \mathrm{Co}$ structure with Co film thickness $N=7$ at temperatures $T_{c}=3.0356$ (a) and $T_{\mathrm{c}} / 2=1.5178$ (b) for evolution from high-temperature initial state.

temperature. Reason for this behavior is connected with $X Y$-type anisotropy, which is realized in $\mathrm{Co} / \mathrm{Cu}(100) / \mathrm{Co}$ structures, and with extremely slow dynamics in twodimensional $X Y$-model characterized by aging not only near the temperature of the Berezinskii-KosterlitzThouless phase transition $T_{\mathrm{BKT}}$, but also in the entire range of the existence of the low-temperature phase [13, 14]. These features of non-equilibrium behavior are conserved in ultrathin $X Y$-type films.

The dependence of aging characteristics on thickness $N$ of ferromagnetic films is demonstrated in Figure 3 where the curves of the autocorrelation function $C\left(t, t_{\mathrm{w}}\right)$ are given for film thicknesses $N=3,5$ at temperatures $T_{c}$ and $T_{\mathrm{c}} / 2$. The analysis of the $C\left(t, t_{\mathrm{w}}\right)$ slowing down with the same waiting times $t_{\mathrm{w}}$ shows that the aging effects in multilayer structure are decreased with increasing thickness of ferromagnetic films $N$ at the critical temperatures $T_{c}(N)$. This is due to the weakening of the correlation for dimensional transition from quasitwo-dimensional systems to three-dimensional bulk systems. The analysis of the $C\left(t, t_{\mathrm{w}}\right)$ curves at $T_{\mathrm{c}} / 2$ in Figure $3 \mathrm{~b}$ indicates the opposite dependence of the autocorrelation function $C\left(t, t_{\mathrm{w}}\right)$ with increasing thickness $N$ of ferromagnetic films in considered range of thickness changes.

It is well known that in the aging regime at $t-t_{\mathrm{w}} \sim$ $t_{\mathrm{w}} \gg 1$ the two-time autocorrelation function is characterized by the scaling form [4]

$$
C\left(t, t_{w}\right) \sim\left(t_{w}\right)^{-b} F_{C}\left(t / t_{w}\right),
$$

where the exponent $b$ at the critical temperature $T_{c}$ is

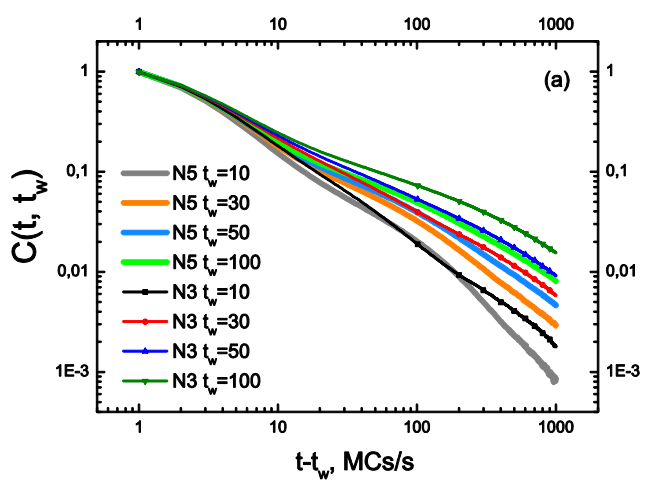

expressed in terms of the critical exponents as $b_{\mathrm{c}}=2 \beta / v z$. The scaling function $F_{c}\left(t / t_{\mathrm{w}}\right)$ is a homogeneous function of its argument $t / t_{\mathrm{w}}$ and, at the long time stage of its evolution with $t-t_{\mathrm{w}}>>t_{\mathrm{w}}>1$, is characterized by the power-law decay

$$
F c\left(t / t_{w}\right) \sim\left(t / t_{w}\right)^{-c_{a}}
$$

where the exponent $c_{a}$ is given at $T_{c}$ by relations $c_{\mathrm{a}}=$ $\mathrm{d} / \mathrm{z}-\theta^{\prime}$ for the evolution from the high-temperature state and by $c_{\mathrm{a}}=1+d / z+\beta / v z$ for the low-temperature one. Here, $\beta, v, z$ and $\theta$ ' are well known static and dynamic critical exponents, $d$ is a system dimension At the thermal bath temperatures $T_{\mathrm{s}} \neq T_{c}$, the exponents $b$ and $c_{a}$ in Eqs. (8) and (9) are not related to the critical exponents.

To check the scaling predictions for $C\left(t, t_{\mathrm{w}}\right)$ given by relation (8), we plot the dependences of $t_{\mathrm{w}}{ }^{2 \beta /(v z)} C\left(t, t_{\mathrm{w}}\right)$ versus $t / t_{\mathrm{w}}$ in Figure 4 for different Co film thicknesses $N$ at the critical temperatures $T_{c}(N)$ with the use of exponent $2 \beta / v z$ chosen to fit the data for different $t_{\mathrm{w}}$ values to a single curve at $t / t_{\mathrm{w}}>>1$. The exponent $b_{\mathrm{c}}=$ $2 \beta / v z$ obtained from this fit is characterized by following values: $2 \beta / v z=0.3184(4)$ for $N=3,2 \beta / v z=0.4140$ (4) for $N=5,2 \beta / v z=0.4364(5)$ for $N=7$, and $2 \beta / v z=0.4562(5)$ for $N=9$. These values of exponent $2 \beta / v z$ demonstrate a dimensional crossover from quasi-two-dimensional to three-dimensional systems.

\section{Conclusions}

A Monte Carlo simulation of the non-equilibrium behavior of multilayer $\mathrm{Co} / \mathrm{Cu}(100) / \mathrm{Co}$ magnetic

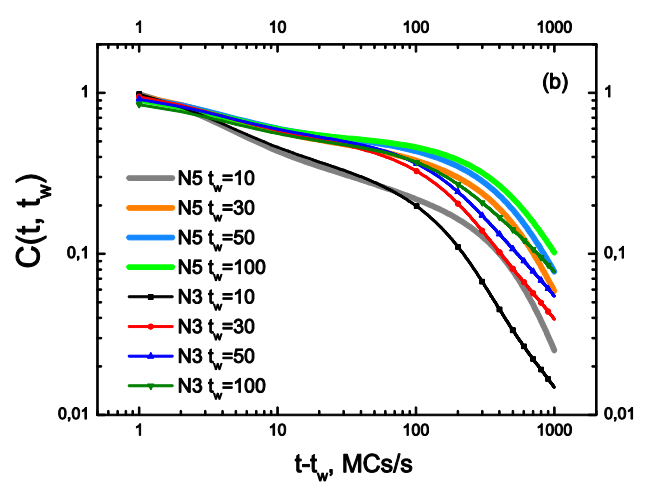

Fig. 3. Time dependence of the autocorrelation function $C\left(t, t_{w}\right)$ as a function $t-t_{w}$ for different values of $t_{\mathrm{w}}$ for $\mathrm{Co} / \mathrm{Cu}(100) / \mathrm{Co}$ structure with Co film thicknesses $N=3,5$ at temperatures $T_{c}$ (a) and $T_{\mathrm{c}} / 2$ (b) for evolution from high-temperature initial state. 

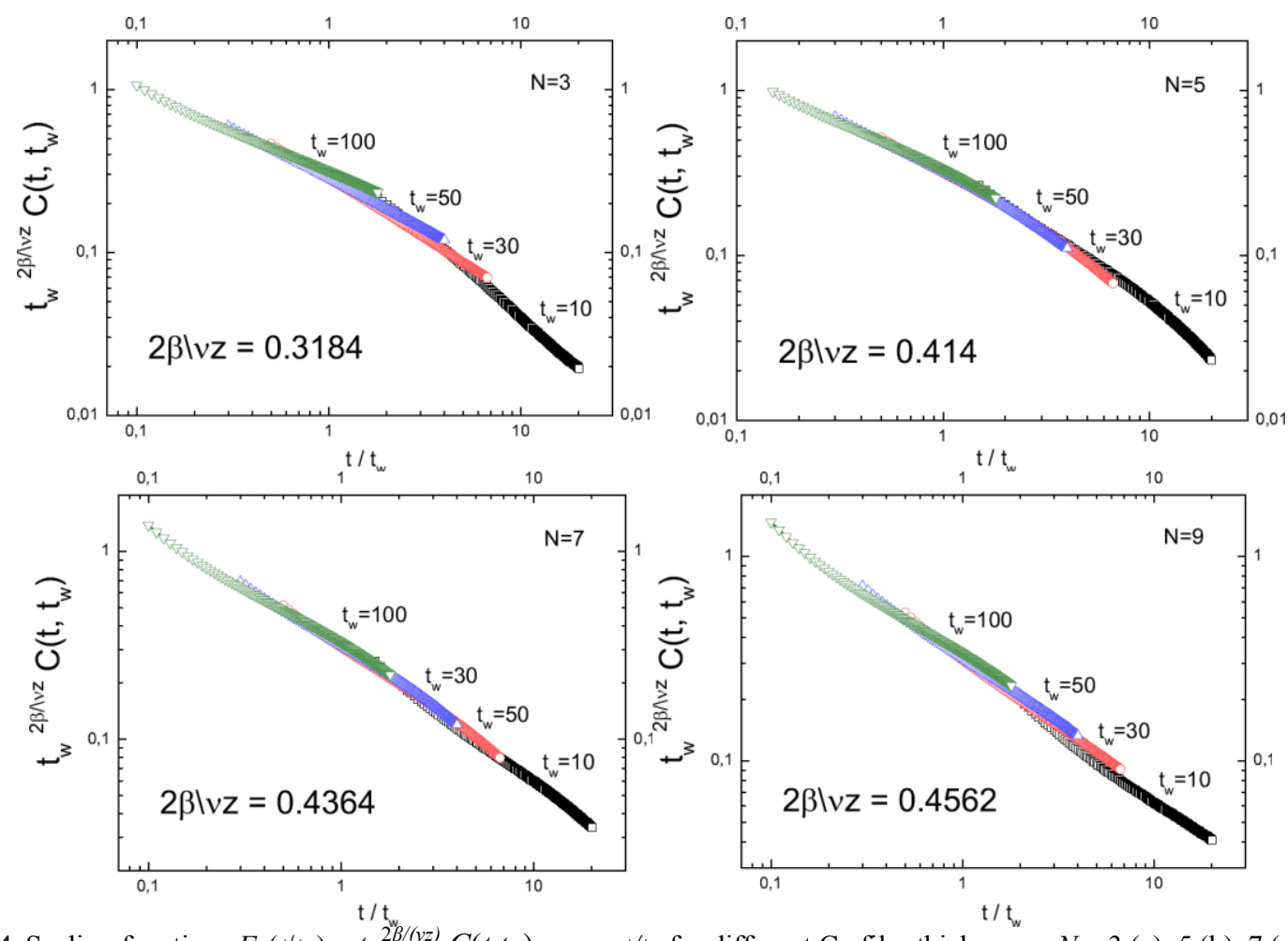

Fig. 4. Scaling functions $F_{\mathrm{C}}\left(t / t_{\mathrm{w}}\right)=t_{\mathrm{w}}{ }^{2 \mathrm{t} /(v z)} C\left(t, t_{\mathrm{w}}\right)$ versus $t / t_{\mathrm{w}}$ for different Co film thicknesses $N=3(\mathrm{a}), 5(\mathrm{~b}), 7$ (c), and 9 (d) at the critical temperatures $T_{c}(N)$ for evolution from high-temperature initial state.

structure was realized with use of the anisotropic Heisenberg model for describing magnetic properties of ultrathin Co films. The calculations of the two-time dependent autocorrelation function $C\left(t, t_{\mathrm{w}}\right)$ revealed the aging phenomena in multilayer structures in wider temperature range at $T \leq T_{\mathrm{c}}$ than in bulk systems by reason of the increase of characteristic spin-spin correlation length in magnetic nanostructures.

The analysis of $C\left(t, t_{\mathrm{w}}\right)$ showed that aging effects are characterized by a slowing down of the correlation time in the system with increasing waiting time $t_{\mathrm{w}}$. It was detected that these effects are increased in the lowtemperature phase in comparison with slowing down of $C\left(t, t_{\mathrm{w}}\right)$ at the critical temperature $T_{\mathrm{c}}$. This phenomenon is connected with extremely slow dynamics in $\mathrm{Co} / \mathrm{Cu}(100) / \mathrm{Co}$ magnetic structures with $\mathrm{Co}$ films as quasi-two-dimensional $X Y$ systems.

The study of dependence of aging characteristics on thickness $N$ of Co films revealed that the aging effects in multilayer structure are decreased with increasing thickness $N$ of ferromagnetic films at the critical temperatures $T_{c}(N)$ but characterized by the opposite dependence at temperatures $T_{c}(N) / 2$.

This work was supported in part by the RFBR through Grant 17-02-00279 and by Grant MD-6024.2016.2 of Russian Federation President. Simulations were carried out on the SKIF-MSU of the Moscow State University and with use of computing resources of the "Applied theoretical physics and parallel computing laboratory" of Omsk State University.

\section{References}

1. Henkel M., Pleimling M., Non Equilibrium Phase Transitions, Vol. 2: Ageing and Dynamical Scaling far from Equilibrium (Theoretical and Mathematical Physics) (Springer, Heidelberg, 2010)
2. J.P. Bouchaud, E. Vincent, J. Hammann, M. Ocio, L.F Cugliandolo, Lect. Notes Phys. 492, 184 (1997)

3. P. Calabrese, A. Gambassi, J. Phys. A 38, R133 (2005)

4. P.V. Prudnikov, V.V. Prudnikov, E.A. Pospelov, P. N. Malyarenko, A.N. Vakilov, Prog. Theor. Exp. Phys. 2015, 053A01 (2015)

5. T. Mukherjee, M. Pleimling, Ch. Binek, Phys. Rev. B 82, 134425 (2010).

6. V.V. Prudnikov, P.V. Prudnikov, A.N. Purtov, M.V. Mamonova, JETP Lett. 104, 776 (2016)

7. V.V. Prudnikov, P.V. Prudnikov, D.E. Romanovskii, JETP Lett. 102, 668 (2015)

8. J. Bass, W.P. Pratt, J. Magn. Magn. Mater. 200, 274 (1999)

9. V.V. Prudnikov, P.V. Prudnikov, D. E. Romanovskiy, J. Phys. D: Appl. Phys. 49, 235002 (2016)

10. P.V. Prudnikov, V.V. Prudnikov, M. A. Medvedeva, JETP Lett. 100, 446 (2014)

11. P.V. Prudnikov, V.V. Prudnikov, M.A. Menshikova, N.I. Piskunova, J. Magn. Magn. Mater. 387, 77 (2015)

12. F. Huang, M.T. Kief, G.J. Mankey, R.F. Willis, Phys. Rev. B. 49, 3962 (1994)

13. L. Berthier, P.C.W. Holdsworth, M. Sellitto, J. Phys. A 34, 1805 (2001)

14. V.V. Prudnikov, P.V. Prudnikov, S.V. Alekseev, I.S. Popov, Phys. Met. Metallogr. 115, 1186 (2014) 